\title{
Assessment of the spectral properties of the double-layer potential matrix $\mathbf{H}$
}

\author{
N. A. Dumont \\ Civil Engineering Department, Pontifical Catholic University of \\ Rio de Janeiro, Brazil
}

\begin{abstract}
The double-layer potential matrix $\mathbf{H}$ of the conventional, collocation boundary element method (CBEM) is singular, as referred to a static problem in a bounded continuum. This means that no rigid body displacements can be transformed between two different reference systems - and that unbalanced forces are to be excluded from a consistent linear algebra contragradient transformation. The properties of $\mathbf{H}^{\mathrm{T}}$ may become quite informative, as they reflect the topology (concavities, notches, cracks, holes) of the discretized domain as well as material non-homogeneities, which comes from the fact that local stress gradients can be represented by fundamental solutions only in a global sense. Symmetries and antisymmetries are also evidenced in $N\left(\mathbf{H}^{\mathrm{T}}\right)$ as well as the spectral properties related to simple polynomial solutions that one may propose as patch tests. In the usual implementations of the CBEM with real fundamental solutions, all eigenvalues $\lambda$ of $\mathbf{H}$ are real, $\lambda \in \mathbb{R}, 0 \geq \lambda<1$ for a bounded domain. This means that $\mathbf{H}$ is a contraction - a paramount mechanical feature that comes up naturally in the frame of a virtual work investigation of Kelvin's (singular) fundamental solution and is resorted to in a simplified variational implementation of the boundary element method.
\end{abstract}

Keywords: boundary elements, hybrid boundary elements, spectral properties, variational methods.

\section{Introduction}

This paper is a counterpart of reference [1], which assesses the single-layer potential matrix G. It in part repeats and in part improves on a recently developed research work [2]. The complete mathematical developments are too extensive 
and demand too many mechanical concepts - as well as elucidative illustrations - to be contained in the present paper, which justifies the preparation of a proper manuscript, to appear soon. The following developments apply to elasticity problems, in general, with results for potential problems inferred directly.

A variationally based counterpart of the boundary element method was introduced in 1987 [3-5]. Starting point was the generalized expression of the total potential energy, to which displacement compatibility assumptions had to be explicitly added, so clarifying the discussion on the symmetry characteristics of the resultant equations: symmetry or nonsymmetry is a matter of adequate or inadequate variational treatment of the boundary conditions. The variationally consistent, generalized displacement formulation thus obtained turned out to be equivalent to the formulation based on the Hellinger-Reissner potential [6], exactly as Pian had developed for finite elements [7]. In allusion to Pian's work this new method was baptized the hybrid boundary element method - HBEM. In fact, the name "hybrid finite element method" had been coined by Pian in 1967 "... to signify elements which maintain either equilibrium or compatibility in the element and then to satisfy compatibility or equilibrium respectively along the interelement boundary" [8]. According to Oden and Reddy [9], Pian has formulated a hybrid displacement method, since it results in a stiffness matrix.

In the HBEM, the null space $N\left(\mathbf{H}^{\mathrm{T}}\right)$ is specifically required, and their properties may turn out difficult to deal with. Owing to their intricacy, it has also become advantageous - and ultimately successful - to develop an alternative variational formulation that only deals with $N(\mathbf{H})[10,11]$. However, the unfolded mathematical properties of $\mathbf{H}^{\mathrm{T}}$ - and how they fit in a variational framework - are elegant and beautiful per se. They are addressed in the following sections starting from a simple virtual work statement. The properties that come out from the present developments may become useful in the evaluation of results also in the frame of the CBEM $[4,12]$. They are presented herein exclusively as linear algebra features, independently from applications that may be derived [13].

\section{Basic assumptions and propositions}

Let an elastic body be submitted to static traction forces $\bar{t}_{i}$ on part $\Gamma_{\sigma}$ of the boundary and to displacements $\bar{u}_{i}$ on the complementary part $\Gamma_{u}$. For the sake of simplicity, body forces are not included. The present formulation applies in principle to a finite, simply-connected open domain $\Omega$ with piece-wise smooth boundary $\Gamma$ that does not have cusps [14]. Unbounded and multiply-connected regions are dealt with later on in terms of the domain $\bar{\Omega}$ defined as the complement of the closure of $\Omega$. Thus, holes are ultimately comprised by the formulation. Internal and edge cracks may also be included in the numerical model provided that ad-hoc functions take the local stress gradients into account [15,16], a subject that is beyond the present scope, although the implications of such topological issues can be addressed in the present context, as they are uncovered as particular properties of $N\left(\mathbf{H}^{\mathrm{T}}\right)$. 
For notation conciseness, the arguments $x, y, z$ of functions in Cartesian coordinates are omitted. Indicial notation is used. A subscript after a comma denotes derivative with respect to the corresponding coordinate direction. Repeated subscripts indicate summation. Arrays of constants are also referred to without subscripts, using bold-face, capital letters for matrices, and bold-face, lower letters for vectors.

\subsection{Proposition on stress and displacement representations}

The numerical model is formulated in terms of two fields:

- One field for displacements on $\Gamma$, given as functions of $n^{d}$ nodal displacement parameters $\mathbf{d}=\left[d_{n}\right] \in \mathbb{R}^{n^{d}}$ located along $\Gamma$. These functions have local support and satisfy the displacement boundary conditions as a premise. This parametric representation is referred to as the external, or displacement, reference system.

- A second field for stresses in $\Omega$, given as a series of functions of $n^{*}$ point force parameters $\mathbf{p}^{*}=\left[p_{m}^{*}\right] \in \mathbb{R}^{n^{*}}$ located along $\Gamma$. These functions have global support and satisfy the domain equilibrium equations as a premise. This parametric representation is referred to as the internal, or force, reference system.

A displacement virtual work principle will provide the conceptual means of correlating these two fields, in a way that naturally leads to the matrix $\mathbf{H}$. Most important, the formulation makes use of $n^{*}=n^{d}$ singular fundamental solutions for the stress representation in $\Omega$. The following proposition is paramount to arrive at the matrix $\mathbf{H}$, as developed both in the CBEM and in the HBEMs, as well as for its adequate mathematical assessment.

Proposition 1 The parameters $\mathbf{d}$ and $\mathbf{p} *$ are identified with nodal displacements and forces that act at the same points distributed along the boundary $\Gamma$, in such a way that $\mathbf{p}^{* \mathrm{~T}} \mathbf{d}$ has the meaning of mechanical work.

There are $n^{\text {ndof }}$ displacement and force degrees of freedom at each nodal point. $n^{n d o f}=1,2$ or 3, for general problems of potential, 2D elasticity and 3D elasticity, respectively. One might in principle develop a formulation with any number of parameters attached to each nodal point [17]. Another possibility would be to have $\mathbf{d}$ and $\mathbf{p}^{*}$ with no clear mechanical meaning at all, just as discretization parameters [18]. Specific problems, such as in the gradient elasticity theory, may demand higher $n^{\text {ndof }}$ for a meaningful numerical discretization.

\subsection{Displacement assumption on $\Gamma$}

The displacements $u_{i}$ are approximated along $\Gamma$ by $u_{i}^{d}$ given as

$$
u_{i}^{d}=u_{i n} d_{n} \quad \text { on } \quad \Gamma
$$

where $u_{i n}$ are polynomial interpolation functions with compact support and $d_{n}$ are nodal displacement parameters. The boundary geometry is approximated from 
the nodal attributes using the same interpolation functions $u_{\text {in }}$ (isoparametric representation).

Equation (1) is supposed to hold along $\Gamma_{u}$, in particular: $\bar{u}_{i}=u_{i n} \bar{d}_{n}$, in which $\bar{d}_{n}$ are nodal values of $\bar{u}_{i}$.

\subsection{Stress assumption in $\Omega$}

The stress field in $\Omega$ is approximated by a sum of homogeneous, fundamental solutions of the domain differential equilibrium equations,

$$
\sigma_{i j}^{*}=\sigma_{i j m}^{*} p_{m}^{*}
$$

where $p_{m}^{*}$ are force parameters and $\sigma_{i j m}^{*}$ are singular fundamental solutions with global support and analytical in $\Omega\left(\sigma_{i j m}^{*}\right.$ may be called, in the present context, interpolation, approximation or trial functions). Although omitted, the arguments of the fundamental solutions are $\left(x-x_{M}, y-y_{M}, z-z_{M}\right)$, where $\left(x_{M}, y_{M}, z_{M}\right)$ is the source point - at which the point force $p_{m}^{*}$ is applied - and $(x, y, z)$ is the field point where the effect of $p_{m}^{*}$ is evaluated. A fundamental solution fulfills following equilibrium equations as a premise:

$$
\left.\begin{array}{l}
\sigma_{j i m, j}^{*}=0 \\
\sigma_{i j m}^{*}=\sigma_{j i m}^{*}
\end{array}\right\} \quad \text { in } \Omega
$$

Except for analyticity, no concern is explicitly made about equilibrium of the fundamental solutions on $\Gamma_{\sigma}$, although some boundary conditions might be included as a premise, too (Green's functions). According to Proposition 1, the fundamental solutions $\sigma_{i j m}^{*}$ turn out to be singular just outside $\Omega$, more precisely at points of application of $p_{m}^{*}$ distributed along $\Gamma$, so that one might express instead of the first line of Eqn (3):

$$
\sigma_{j i m, j}^{*}+\Delta_{i m}=0 \quad \text { in } \quad \Omega_{e x t}=\Omega \cup \Omega_{0}
$$

This is the usual expression found in the literature on boundary integral equations, except that, for clarification of concepts, the extended domain $\Omega_{e x t}=\Omega \cup \Omega_{0}$ is also used, where $\Omega_{0}$ is understood as a set of infinitesimally small, closed regions containing each point of singularity. $\Delta_{i m}$ is a pulse function, which has zero value everywhere in the domain, except for the vicinity of the point of application of $p_{m}^{*}$, where it tends to infinity.

Proposition 2 Further to Proposition 1, let $\Delta_{i m}$ be normalized, for a domain $\Omega_{\text {ext }}$ comprising a singularity, as

$$
\int_{\Omega_{e x t}} \Delta_{i m} \mathrm{~d} \Omega \equiv \int_{\Omega_{0}} \Delta_{i m} \mathrm{~d} \Omega=\delta_{i m}
$$

where $\delta_{i m}$ is a generalized Kronecker delta. 
Thus, $p_{m}^{*}$ in Eqn (2) has the meaning of a unit point force applied at a nodal point on $\Gamma$, according to Proposition 1 , with $m$ characterizing both location and direction.

$\Omega$ is the actual domain of interest and there is in principle no need to invoke the extension $\Omega_{e x t}$. The singularity explicitly expressed by $\Delta_{i m}$ in Eqn (4) is a welcome feature, as it assures that the resulting equation systems are well conditioned.

\section{Displacement virtual work}

In the absence of body forces, equilibrium of the stress field $\sigma_{i j}^{*}$ with forces $\bar{t}_{i}$ along $\Gamma_{\sigma}$ occurs if and only if

$$
\int_{\Omega} \sigma_{j i}^{*} \delta \epsilon_{i j}^{d} \mathrm{~d} \Omega=\int_{\Gamma_{\sigma}} \bar{t}_{i} \delta u_{i}^{d} \mathrm{~d} \Gamma
$$

for an arbitrary, virtual field of displacements $\delta u_{i}^{d}$ such that $\delta u_{i}^{d}=0$ on $\Gamma_{u}$ and $\delta \epsilon_{i j}^{d}=\frac{1}{2}\left(\delta u_{i, j}^{d}+\delta u_{j, i}^{d}\right)$ in $\Omega$. The stress field $\sigma_{i j}^{*}$ approximated according to Eqn (2) already satisfies Eqn (3). Taking into account that $\delta u_{i}^{d}=0$ on $\Gamma_{u}$, the boundary integral of Eqn (6) may be extended to the whole boundary $\Gamma$. Integration by parts of the term on the left-hand side of Eqn (6), application of Green's theorem and use of Eqns (1) and (2) lead to the matrix expression

$$
\mathbf{H}^{\mathrm{T}} \mathbf{p}^{*}=\mathbf{p}
$$

where $\mathbf{H}=\left[H_{m n}\right] \in \mathbb{R}^{n^{*} \times n^{d}}$ and $\mathbf{p}=\left[p_{n}\right] \in \mathbb{R}^{n^{d}}$ are expressed, for a bounded domain, as

$$
\begin{gathered}
H_{m n}=\int_{\Gamma} \sigma_{j i m}^{*} \eta_{j} u_{i n} \mathrm{~d} \Gamma \equiv \int_{\Gamma_{e x t}} \sigma_{j i m}^{*} \eta_{j} u_{i n} \mathrm{~d} \Gamma+\delta_{m n} \\
\equiv \int_{\Gamma_{f p}} \sigma_{j i m}^{*} \eta_{j} u_{i n} \mathrm{~d} \Gamma+\int_{\Gamma_{d i s c}} \sigma_{j n m}^{*} \eta_{j} \mathrm{~d} \Gamma \\
p_{n}=\int_{\Gamma} t_{i} u_{i n} \mathrm{~d} \Gamma
\end{gathered}
$$

$\mathbf{p}$ is the vector of equivalent nodal forces, which is in part known (on $\Gamma_{\sigma}$ ) and must be in part evaluated (as reaction forces along $\Gamma_{u}$ ), for a general mixed boundary problem. $\mathbf{H}$ is the same double-layer potential matrix obtained in the collocation boundary element method [19]. $\delta_{m n}$ is the identity matrix I of order $n^{*}=n^{d}$. The evaluation of $\mathbf{H}$ is straightforward also for unbounded and multiplyconnected domains, with the boundary unit normal $\vec{\eta}$ always pointing outward. The boundary integral of Eqn (8) is singular, for $m$ and $n$ referring to the same nodal point, but may be completely evaluated either mathematically - split into a Cauchy principal value (in terms of a finite-part integral) and a discontinuous term - or by using rigid body displacements, according to Eqn (13). Figure 1 illustrates the three mathematical equivalences stated in Eqn (8) for the boundary segment in the vicinity of a singularity point, in which it is seen that the singularity is outside 
the domain $\Omega$. Application of the virtual work principle of Eqn (6) to an unbounded domain is straightforward. Figure 2 illustrates Eqn (8) applied to an infinitely large domain $\bar{\Omega}$ - complementary to the closure of $\Omega$ - enclosed externally by a single boundary $\Gamma_{\infty}$ and internally by the same boundary of Fig. 1, but characterized as $\bar{\Gamma}$, since the outward normal $\vec{\eta}$ is reversed. The finite-part integrals for $\Gamma$ and $\bar{\Gamma}$ have the same magnitude and reversed signals. The discontinuous terms for $\Gamma$ and $\bar{\Gamma}$ add to $\delta_{i m}$, as a result of Eqn (5). Then, if one denotes the result of Eqn (8) for the complementary domain $\bar{\Omega}$ as $\overline{\mathbf{H}}$, a corresponding expression may be written as

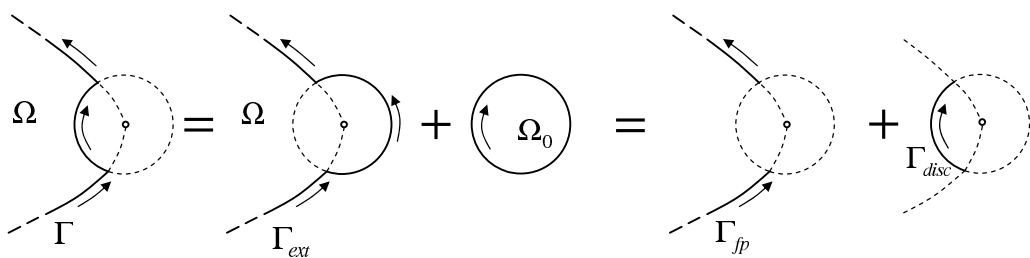

Figure 1: Illustration of the three mathematically equivalent forms of Eqn (8) for a bounded domain.

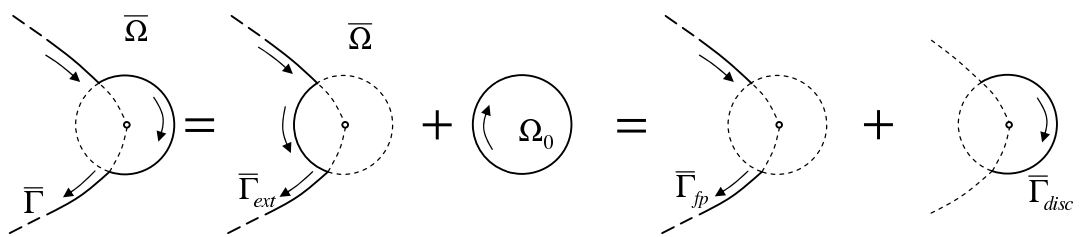

Figure 2: Illustration of the three mathematically equivalent forms of Eqn (8) for an unbounded domain.

$$
\overline{\mathbf{H}}^{\mathrm{T}} \mathbf{p}^{*}=\overline{\mathbf{p}} \quad \text { where } \quad \overline{\mathbf{H}}^{\mathrm{T}}=\mathbf{I}-\mathbf{H}^{\mathrm{T}}
$$

The coefficients of $\overline{\mathbf{p}}$ for prescribed tractions are evaluated along $\bar{\Gamma}_{\sigma}$.

\section{Linear algebra properties of the transformation matrix $H$}

As formulated, $\mathbf{H}^{\mathrm{T}}$ is an equilibrium matrix that transforms nodal forces $\mathbf{p}^{*}$ of the internal reference system into equivalent nodal forces $\mathbf{p}$ of the external reference system. It is obtained in the complete frame of the Hellinger-Reissner potential that $\mathbf{H}$ is a kinematic matrix that transforms nodal displacements $\mathbf{d}$ of the external system into equivalent nodal displacements $\mathbf{d}^{*}$ of the internal system. This is 
expressed as

$$
\mathbf{d}^{*}=\mathbf{H d} \quad \Leftrightarrow \quad \mathbf{p}=\mathbf{H}^{\mathrm{T}} \mathbf{p}^{*} ; \quad \overline{\mathbf{d}}^{*}=\overline{\mathbf{H}} \mathbf{d} \quad \Leftrightarrow \quad \overline{\mathbf{p}}=\overline{\mathbf{H}}^{\mathrm{T}} \mathbf{p}^{*}
$$

and it is obtained from Eqns (10) and (11) that

$$
\mathbf{d}^{*}+\overline{\mathbf{d}}^{*}=\mathbf{d} \text { and } \mathbf{p}+\overline{\mathbf{p}}=\mathbf{p}^{*}
$$

Let the columns of the matrix $\mathbf{W} \equiv N(\mathbf{H})=\left[W_{n s}\right] \in \mathbb{R}^{n^{d} \times n^{\text {rig }}}$ be a basis of the set of $n^{r i g}$ rigid body displacements that the bounded body may suffer, as measured at the nodal points. $\mathbf{W}$ depends only on the body geometry. Also, define a matrix $\mathbf{V} \equiv N\left(\mathbf{H}^{\mathrm{T}}\right)=\left[V_{n s}\right] \in \mathbb{R}^{n^{d} \times n^{r i g}}$. Differently from $\mathbf{W}, \mathbf{V}$, as expressed in the following, depends on $u_{i n}$ and $\sigma_{i j m}^{*}$ (and thus from the material properties of the elastic problem [20]):

$$
\begin{aligned}
& \mathbf{H W}=\mathbf{0} ; \quad \mathbf{H}^{\mathrm{T}} \mathbf{V}=\mathbf{0} \\
& \overline{\mathbf{H}} \mathbf{W}=\mathbf{W} ; \quad \overline{\mathbf{H}}^{\mathrm{T}} \mathbf{V}=\mathbf{V}
\end{aligned}
$$

\subsection{Some basic theorems}

Owing to space restrictions, following theorems are given without proof. These and a few more theorems are detailed in the full version of the paper.

Theorem 1 (Evaluation of $\mathbf{V}$ ) If $\mathbf{H}$ is nondefective, then $\mathbf{W}^{\mathrm{T}} \mathbf{V}, \mathbf{H}+\mathbf{W} \mathbf{W}^{\mathrm{T}}$ and $\mathbf{H}+\mathbf{W W}^{\mathrm{T}}$ have all full range. Then, a simple means of evaluating $\mathbf{V}$, whenever required, is by solving the equation system $\left(\mathbf{H}+\mathbf{W W}^{\mathrm{T}}\right) \mathbf{V}=\mathbf{W}$.

Theorem $\mathbf{2}$ (Contraction) $\mathbf{H}$ is a contraction [21], and so is $\mathbf{H}^{\mathrm{T}}$, since from Eqns (11) and (12), for any norm $\|\bullet\|,\|\mathbf{p}\|=\left\|\mathbf{H}^{\mathrm{T}} \mathbf{p}^{*}\right\| \leq\left\|\mathbf{p}^{*}\right\|, \quad\|\overline{\mathbf{p}}\|=$ $\left\|\overline{\mathbf{H}}^{\mathrm{T}} \mathbf{p}^{*}\right\| \leq\left\|\mathbf{p}^{*}\right\|$.

Theorem 3 (Diagonal of H) $0<H_{j j}<1, \quad 0<\bar{H}_{j j}<1 \quad$ for all $j=$ $1, \cdots, n^{*}=n^{d}$.

Theorem 4 (Positive direction) $\mathbf{p}^{* \mathrm{~T}} \mathbf{p}^{*}>\mathbf{p}^{* \mathrm{~T}} \mathbf{p} \geq 0, \quad \mathbf{p}^{* \mathrm{~T}} \mathbf{p}^{*} \geq \mathbf{p}^{* \mathrm{~T}} \overline{\mathbf{p}}>0$.

Theorem 5 (Real eigenvalues) All eigenvalues $\lambda$ and eigenvectors $\phi$ of $\mathbf{H}$ are real. Moreover, for any $\lambda$ : $0 \leq \lambda<1$.

Theorem 4 follows from Proposition 1 and the displacement and stress assumptions, or simply from Theorem 3. A proof of Theorem 5 follows from Theorem 4, which must be valid for any eigenvector $\phi$ of $\mathbf{H}^{\mathrm{T}}$. Suppose that $\phi$ is complex, with corresponding complex eigenvalue $\lambda$, and let ( )* only in the following outline mean the Hermitian adjoint. One obtains from $\boldsymbol{\phi}^{*} \mathbf{H}^{\mathrm{T}} \boldsymbol{\phi} \geq 0$ and Theorem 2 that $0 \leq \Re(\lambda)<1$. The proof that $\Im(\lambda)=0$ deserves more attention. A strict proof takes into account some degenerate cases as when geometry symmetries are included in the formulation. 
In case of Neumann boundary conditions, the complete solution of a problem (except for rigid body displacements) is obtained by solving Eqn (10) for an unbounded domain, as $\overline{\mathbf{H}}^{\mathrm{T}}$ is nonsingular, or Eqn (7) for a bounded domain, subjected to the restriction that $\mathbf{V}^{\mathrm{T}} \mathbf{p}^{*}=0$ (see also [2]).

Given a submatrix $\mathbf{W}_{N}=\left[W_{N n s}\right] \in \mathbb{R}^{n^{n d o f} \times n^{r i g}}$ of $\mathbf{W}$ related to a nodal point $N$, then $\mathbf{W}_{N}^{\mathrm{T}} \mathbf{W}_{N}$ is always nonsingular and well conditioned, independently from problem topology and from material properties. Then, $\mathbf{H W}=\mathbf{0}$, Eqn (13), for a bounded domain is a means to evaluate the coefficients of $\mathbf{H}$ about its main diagonal, thus circumventing the need to deal with the singular boundary integral of Eqn (8) - which is actually a not palpable advantage for large equation systems. A similar property is attached to $\mathbf{V}$, although involving some far reaching concepts and being not as generally applicable [20].

Theorem 6 If a numerical model converges to the idealized mechanical problem with increasing mesh refinement, then it is possible to have a boundary mesh such that, for all points in $\Omega$ not too close to the boundary nodes, $\left\|\sigma_{i j m}^{*} V_{m r}\right\| \leq \epsilon$ for an arbitrarily small error $\epsilon \geq 0$.

This theorem [13] follows from the fact that $\mathbf{V}$ is a basis of point forces $\mathbf{p}^{*}$ that are not in equilibrium and therefore cannot generate a stress state [4]. This theorem may be used in a reverse way, according to the following.

Proposition 3 For points in $\Omega$ that are too close to a boundary node, so that the boundary layer effect becomes perceptible (or at the boundary node itself, which is actually outside $\Omega$, as illustrated in Figs. 1 and 2), the term $\sigma_{i j m}^{*}$ of the fundamental solution in Eqn (2) that tends to increase without bounds may be consistently replaced with finite terms evaluated in such a way that $\left\|\sigma_{i j m}^{*} V_{m r}\right\|=$ minimum, for elasticity problems in general.

Let $\mathbf{V}_{N}=\left[V_{N n s}\right] \in \mathbb{R}^{n^{n d o f} \times n^{r i g}}$ be a submatrix of $\mathbf{V}$ related to a nodal point $N$. The use of Proposition 3 to obtain results at a point close to or at the nodal point $N$ in terms of least squares is only possible if $\mathbf{V}_{N}^{\mathrm{T}} \mathbf{V}_{N}$ is well conditioned. However, this is not the case for points close to or at crack or notch tips, strong concavities and cavities, as well as for symmetry axes or planes in case of numerical problems that have symmetries embedded in the formulation, not to mention some unpredictable locations on a geometrically convex domain that contains a non-homogeneous material [20]. In fact, a problem in a convex domain with non-homogeneous properties may be expressed via a Kirchhoff-like transform as an equivalent one with homogeneous properties: the transformed geometry may present strong concavities. It is amazing to find out that the problem formulated from the proposition that $\left\|\sigma_{i j m}^{*} V_{m r}\right\|$ is a minimum is always consistent, but involves, in case of strong local non-convexities, a division between small numbers that are in the range of the discretization error, which still validates Theorem 6 but then becomes of no usage for a practical implementation [20].

The explanation of this fact is extremely simple, although its has taken many years of investigation to become clear. For points in a region far from the boundary or close to a strictly convex part of the boundary, the stress gradient 
may be globally described and Proposition 3 is, whenever necessary, a means of eliminating the spurious gradient locally introduced by the singular fundamental solution. On the other hand, a region close to crack or notch tips, for instance, has a stress gradient that is locally conditioned and must be described adequately. In such a case the ill-conditioning of $\mathbf{V}_{N}^{\mathrm{T}} \mathbf{V}_{N}$ only tells that the mathematics of the problem is not contradicting the local mechanical peculiarities.

This problem has astonished the author and his collaborators from the very beginning of the development of the HBEM. However, it has been completely understood and explored in its mathematical elegance and appropriateness. Depending on the problem, the local stress gradient must be dealt with directly. There are in general sound mechanical alternatives to circumvent ill-conditioning problems related to $\mathbf{V}_{N}^{\mathrm{T}} \mathbf{V}_{N}[13,22]$.

As shown in the extended version of this paper, the spectrum $\sigma(\mathbf{H})$ characterizes if a domain is bounded or unbounded, simply or multiply connected. Together with $\left\|\mathbf{V}_{N}^{\mathrm{T}} \mathbf{V}_{N}\right\|$, for $N$ spanning all the boundary nodal points of the numerical model, cavities and concavities may be precisely identified, in general, as well as embedded symmetries.

\section{Conclusions}

This paper emphasizes the conceptual basis of the double-layer potential matrix $\mathbf{H}$, as derived from a well established variational principle and directly applicable to the conventional boundary element method. Moreover, its most elementary linear algebra properties are investigated. However, owing to paper length constraint several features had to be omitted and their discussion postponed to a more extensive publication. The most important feature that could not be investigated is related to the eigenvalues of $\mathbf{H}$ as a contraction. The main advantages of the CBEM, as usually implemented, rely on these linear algebra properties. On the other hand, a boundary element formulation - variational or not might be conceived, in which the boundary interpolation functions $u_{i n}$ or the singular fundamental solutions $\sigma_{i j m}^{*}$ are not according to the basic equations and propositions of Section 2. A feasible boundary element formulation (in which $\mathbf{H}$ is defective, for instance) might still be developed, although lacking generality and several properties that render the equations well conditioned. The "regularized boundary element methods", in which the source point is located at a finite distance outside the domain, are just an example of formulation for which the matrix $\mathbf{H}$ has no clear and sound properties. The mathematical properties, for the problem as stated, owe their elegance to two in part related singularities: of the fundamental solutions and in terms of linear algebra. Not coincidentally, the single-layer potential matrix $\mathbf{G}$ of the boundary element methods also features interesting linear algebra properties that still deserve a deeper investigation [1].

\section{Acknowledgements}

This project was supported by the Brazilian agencies CAPES, CNPq and FAPERJ. 


\section{References}

[1] Dumont, N.A., An assessment of the spectral properties of the matrix G used in the boundary element methods. Computational Mechanics, 22, pp. 3241, 1998.

[2] Dumont, N.A., On the spectral properties of the double layer potential matrix $\mathrm{H}$ of the boundary element methods. PACAM XI - 11th Pan-American Congress of Applied Mechanics, ed. A.R. Aguiar, Foz do Iguaçu.

[3] Dumont, N.A., The hybrid boundary element method. Boundary Elements IX, ed. C.A. Brebbia; W. Wendland and G. Kuhn, vol. 1, Mathematical and Computational Aspects. Computational Mechanics Publications (Southampton) and Springer: pp. 125-138, 1987.

[4] Dumont, N.A., The hybrid boundary element method: An alliance between mechanical consistency and simplicity. Applied Mechanics Reviews, 42(11, part 2), pp. S54-S63, 1989.

[5] Dumont, N.A., Variationally-based hybrid boundary element methods. Computer Assisted Mechanics and Engineering Sciences, (10), pp. 407-430, 2003.

[6] Reissner, E., On a variational theorem in elasticity. Journal of Mathematical Physics, 29, pp. 90-95, 1950.

[7] Pian, T.H.H., Derivation of element stiffness matrices by assumed stress distribution. AIAA Journal, 2, pp. 1333-1336, 1964.

[8] Pian, T.H.H., Reflections and remarks on hybrid and mixed finite element methods. Hybrid and Mixed Finite Element Methods, ed. A.N. Atluri; R.H. Gallagher; O.C. Zienkiewicz. John Wiley \& Sons, pp. 565-570, 1983.

[9] Oden, J.T. \& Reddy, J.N., An Introduction to the Mathematical Theory of Finite Elements. John Wiley \& Sons: New York, 1976.

[10] Dumont, N.A. \& Aguilar, C.A., The best of two worlds: The expedite boundary element method. Engineering Structures, 43, pp. 235-244, 2012.

[11] Aguilar, C.A., An expedite implementation of the hybrid boundary element method for potential and elasticity problems. Ph.D. Thesis (in Portuguese), Pontifical Catholic University of Rio de Janeiro, Brazil, 2013.

[12] Gaul, L., Wagner, M. \& Wenzel, W., Efficient field point evaluation by combined direct and hybrid boundary element methods. Engineering Analysis with Boundary Elements, 21, pp. 215-222, 1998.

[13] Dumont, N.A., Fundamentals of the hybrid boundary element method. Engineering Analysis with Boundary Elements, 2013. To be submitted.

[14] Chen, C. \& Zhou, J., Boundary Element Methods. Academic Press: London, 1992.

[15] Dumont, N.A. \& Lopes, A.A.O., On the explicit evaluation of stress intensity factors in the hybrid boundary element method. Fatigue \& Fracture of Engineering Materials \& Structures, 26, pp. 151-165, 2003.

[16] Dumont, N.A. \& Mamani, E.Y., Generalized Westergaard stress functions as fundamental solutions. Computer Modeling in Engineering \& Sciences, 78(2), pp. 109-150, 2011. 
[17] Tomlinson, K., Pullan, A. \& Bradley, C., Selecting a derivative boundary element formulation for use with Hermitian interpolation. Computational Mechanics'95, ed. S.N. Atluri; G. Yagawa and T.A. Cruse. Springer: Heidelberg, volume 2, pp. 2648-2653, 1995.

[18] Dumont, N.A. \& Prazeres, P.G.C., Hybrid dynamic finite element families for the general analysis of time-dependent problems. Procs. ICSSD 2005 Third International Conference on Structural Stability and Dynamics, eds. Unnikrishnan, Reddy \& Chandra, Florida, USA, 10 pp (on CD), 2005.

[19] Brebbia, C.A., Telles, J.C.F. \& Wrobel, L.C., Boundary element techniques: theory and applications in engineering. Prentice Hall: Berlin, 1984.

[20] Dumont, N.A., Topological aspects in the equilibrium-based implementation of finite/boundary element methods for FGMs. FGM 2006 - Multiscale and Functionally Graded Materials Conference 2006. American Institute of Physics: Honolulu, Hawaii, pp. 658-663, 2008.

[21] Ben-Israel, A. \& Greville, T.N.E., Generalized inverses: theory and applications. R.E. Krieger: Huntington, 1980.

[22] Oliveira, M.F.F. \& Dumont, N.A., Conceptual completion of the simplified hybrid boundary element method. International Conference on Boundary Element Techniques, eds. E.J. Sapountzakis \& F.M. Aliabadi. EC Ltd.: UK, pp. 49-54, 2009. 